\title{
A Cellular Automaton Model for Traffic Flow - Investigating the Effect of Turning
}

\author{
Tracy Finner ${ }^{\dagger}$ and Matthew A. Beauregard ${ }^{\ddagger}$ \\ ${ }^{\dagger}$ Department of Mathematics, University of Arizona, $A Z$ \\ ${ }^{*}$ Department of Mathematics, Baylor University, Waco, TX \\ *Mentor: beauregama@sfasu.edu
}

\begin{abstract}
A cellular automaton model is proposed, modeling vehicular traffic flow on a two dimensional lattice in which the vehicles turn at an intersection with a given probability. It is shown that the introduction of turning reduces the long-term average velocity, and can be predicted by a power law depending on the probability of a vehicle turning and the density of cars. The reduction in speed decreases rapidly once the light cycle length surpasses a certain threshold, the value of which can be predicted from the observed power law.
\end{abstract}

\section{KEYWORDS}

cellular automaton, traffic flow, traffic light strategy, turning, dynamical systems, power law

\section{INTRODUCTION}

It is an everyday occurrence in North America to be influenced by traffic. In many cities congestion of traffic is attenuated by the introduction of highways or multiple lanes increasing the overall capacity of vehicles a network of roads can carry. However this is not a tractable solution for many cities that do not have affordable space to accommodate such an improvement. Hence there is a need to optimize traffic flow given the current network of roads through speed limits, roundabouts, one-way traffic, and most importantly, choosing the appropriate lighting strategy. Still, these alternatives require a large capital investment which should be done without adequate study of the effect these changes will have on the overall vehicular flow. Experimentation is not only costly but may lead to undesirable results. A more cost-effective approach is to study each effect through realistic mathematical modeling that incorporates probabilistic features of human behavior.

Traffic flow can either be studied macroscopically, mesoscopically, or microscopically (see Kerner ${ }^{10}$ for a complete exposition of approaches). Macroscopic models have been studied by many authors. $1,2,4,9,11,13$ A typical approach in the macroscopic model is to view the vehicles as a continuum and model them similar to that of a one-dimensional compressible fluid. The conservation of mass and momentum take the form of first order partial differential equations with the density and velocity of the vehicles serving as unknowns. In the mesoscopic or kinetic models, originally introduced by Prigogine, ${ }^{14}$ the governing equations are based on the Boltzman type kinetic equations. In these models a relaxation term is introduced in combination with a slowing down term to describe interactions due to braking of vehicles.

The cellular automaton (CA) models have been designed to study traffic flow at the microscopic level and are the basis of the model used in this paper. Each vehicle is viewed as a particle far from equilibrium and updated in parallel according to certain rules. The interactions of the vehicles can be complex, thus the particular effect of cars turning onto cross streets is examined in this paper. The authors recommend Chowdhury ${ }^{6}$ and Gartner and Stamatiadis ${ }^{8}$ for an overview of current CA models under study, and Chowdhury ${ }^{7}$ for a more thorough review. In this paper we introduce an extension to the Biham-Middleton-Levine $(\mathrm{BML})^{3}$ model and investigate the effect a turning 
vehicle has on the optimal light cycle length for a synchronous lighting strategy at a particular density. The reduction in the average velocity can be predicted using a newly developed power law.

The paper is organized as follows. The traffic model is introduced in the next section. Integrating turning necessitates a greater interaction among the vehicles as in previous models. Results of the model are given in section 3. It is observed that the flow is reduced as a consequence of turning vehicles and reduction in the average velocity can be predicted using a power law that depends on the density of cars and the turning velocity. Concluding remarks are given in section 4 .

\section{CELLULAR AUTOMATON MODEL}

The model presented uses a two-dimensional square lattice where each cell can either be empty or occupied by a vehicle traveling east or north. The authors consider single, one-way traffic lanes without allowing the overtaking of vehicles. Two-way traffic flow is not considered. There exists a traffic signal at each intersection of north and eastbound lanes and the behavior (green or red) is controlled by a global synchronized light strategy. In this strategy the traffic light is uniform in each direction, for example, green lights for all eastbound traffic simultaneously, then switched to a red light for all eastbound traffic, and green to northbound streets. This greatly simplifies the ability to investigate the dynamics as periodic boundary conditions reduce the lattice of roads to the case of one eastbound and northbound intersection. The orientation of the light is switched at a user-specified time step, $T$, herein referred as the light cycle length.

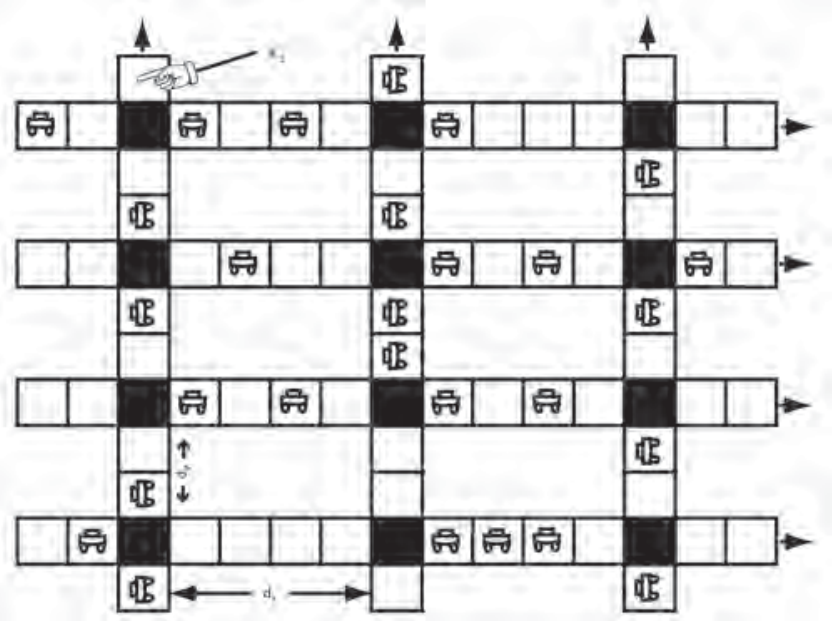

Figure 1. The vehicles are initially assigned random positions and velocities. The vehicles are evenly distributed among the roads. At each intersection a light exists that is either green or red for the oncoming eastbound or northbound traffic. The positions of the vehicles are associated with a particular cell's location $a_{i j}$. Parameters used: $N_{x}=4, N_{y}=3, N_{v}=28, d_{x}=4, d_{y}=2$.

There are $N_{x}$ and $N_{y}$ eastbound and northbound lanes, respectively. The total number of cars, $N_{v}$, are evenly distributed among the eastbound and northbound lanes. Periodic boundary conditions at the edge of the lattice are considered, hence the value of $N_{v}$ is conserved. The density of cars on a given lattice is

$$
\rho=N_{v} /\left(N_{x} N_{y}\left(d_{x}+d_{y}+1\right)\right)
$$

where $d_{x}$ and $d_{y}$ are the number of cells between northbound and eastbound lanes, respectively, and the denominator is the total capacity of the network. Each cell's location is stored in a sparse position matrix $a_{i j}$. For illustrative purposes the lattice for $N_{x}=4, N_{y}=3, N_{v}=28, d_{x}=4$, and $d_{y}=2$ is shown in Fig. 1.

\section{AJUR volume 12 | Issue 1 | August 2014}


The value $d_{n}$ is the distance between vehicles $x_{n}$ and $x_{n+1}$ on the same road, while the value $\widetilde{d}_{n}$ is the distance to the next light plus the distance to the next vehicle on the next road perpendicular to the current one. If $d_{n}$ is one then the next vehicle is in the next adjacent cell. The distance from the current vehicle to the next intersection is given by $s_{n}$. An illustrative cartoon is shown in Fig. 2, in which $d_{n}=3, \widetilde{d}_{n}=4$, and $s_{n}=2$.

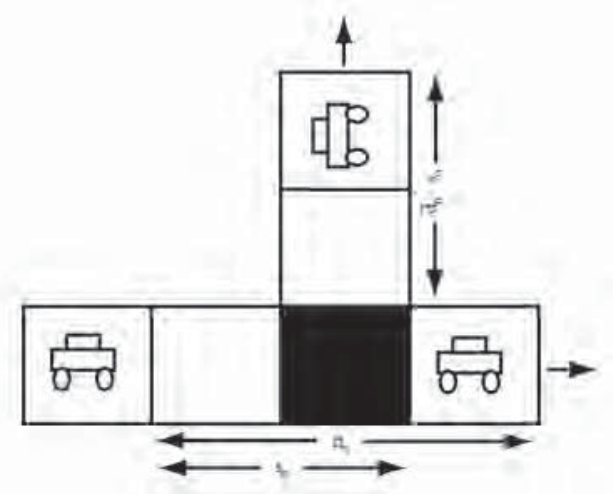

Figure 2. A schematic of vehicles near an intersection. The coordinates of each car are stored in a sparse matrix $a_{i j}$ which makes the calculations easier to compute.

The indices $i$ and $j$ refer to the cell location which is mapped to the vehicle $x_{n}$.

Each vehicle's velocity takes on discrete values of $0,1,2, \ldots, v_{\max }$. The vehicles are initially assigned random positions and velocities. No vehicle is initially placed in an intersection and no more than one vehicle may occupy a cell. The vehicle's positions and velocities are updated in parallel by applying the following rules at each time step:

Step 1: Acceleration

$$
v n \rightarrow \min \left(v_{n}+1, v_{\max }\right) .
$$

Step 2: Traffic Light, Turning, Collision Prevention

Case 1: IF INTERSECTION FLAG ON, i.e. CAR IN INTERSECTION (Turn FLAG OFF)

(a) If traffic light is red in front of $n^{\text {th }}$ vehicle then

$$
v_{n} \rightarrow \min \left(v_{n}, v_{\text {turn }}, d_{n}-1, s_{n}-1\right)
$$

(b) Otherwise traffic light is green in front of $n^{\text {th }}$ vehicle

i. If the next two cells directly past next intersection are occupied then

$$
v_{n} \rightarrow \min \left(v_{n}, v_{\text {turn }}, d_{n}-1, s_{n}-1\right)
$$

ii. Otherwise

$$
v_{n} \rightarrow \min \left(v_{n}, v_{\text {turn }}, d_{n}-1\right)
$$




\section{Case 2: IF INTERSECTION FLAG OFF}

(a) Random turning with probability $p_{\text {turn }}$

i. If traffic light is red in front of $n^{\text {th }}$ vehicle then

$$
v_{n} \rightarrow \min \left(v_{n}, d_{n}-1, s_{n}-1\right) .
$$

ii. Otherwise traffic light is green in front of $n^{\text {th }}$ vehicle

A. If $s_{n} \geq v_{\text {turn }}$ then $v_{n} \rightarrow \min \left(v_{n}, d_{n}-1, s_{n}\right)$

- If updated velocity $v_{n}=s_{n}$ then INTERSECTION FLAG ON

B. Otherwise $s_{n}<v_{\text {turn }}$ then $v_{n} \rightarrow \min \left(v_{n}, v_{\text {turn }}, d_{n}-1, \widetilde{d}_{n}-1\right)$.

- If updated velocity $v_{n}>s_{n}$ then TURNING FLAG ON

(b) Car not selected for turning

i. If traffic light is red in front of $n^{\text {th }}$ vehicle then

$$
v_{n} \rightarrow \min \left(v_{n}, d_{n}-1, s_{n}-1\right) .
$$

ii. Otherwise traffic light is green in front of $n^{\text {th }}$ vehicle

A. If the next two cells directly past next intersection are occupied then

$$
v_{n} \rightarrow \min \left(v_{n}, d_{n}-1, s_{n}-1\right) .
$$

B. Otherwise

$$
v_{n} \rightarrow \min \left(v_{n}, d_{n}-1\right) .
$$

Step 3: Random slowdown with probability $p_{\text {slow }}$

$$
v_{n}=\max \left(v_{n}-1,0\right) .
$$

Step 4: Update Position

Case 1: IF TURNING FLAG ON (Turn FLAG OFF)

(a) If Eastbound turning to Northbound lane then

$$
\mu=i+s_{n}, v=j-\left(v_{n}-s_{n}\right)
$$

(b) Otherwise Northbound turning to Eastbound lane

$$
\mu=i+\left(v_{n}-s_{n}\right), v=j+s_{n}
$$

Case 2: IF TURNING FLAG OFF

(a) If Eastbound vehicle then

$$
\mu=i, v=j+v_{n}
$$

(b) Otherwise Northbound vehicle then

$$
\mu=i-v_{n}, v=i
$$

Update Position: $a_{i j} \rightarrow a_{\mu v}$. 
In step 1 each vehicle is allowed to accelerate provided it is not at the maximum velocity. This refers to the natural human tendency to want to drive as fast as allowed. Speed is incremented by one at each time step that the car is allowed to accelerate.

Step 2 contains the primary interaction among the vehicles. This step is very similar to that found in the algorithm in Brockfeld et al., ${ }^{5}$ differing in that each vehicle can be selected for turning with a probability of $p_{\text {turn }}$. Turning only begins if the next traffic light is green. In this situation, the vehicle's speed is taken to be the minimum of its current velocity, the user-prescribed turning velocity $v_{\text {turn }}$, the distance to the next vehicle on the current road, or the distance to the next vehicle on the road it will be turning on to. Once a car, $x_{n}$, at position $a_{i j}$ is selected to turn, there are three distinct situations which can occur.

First, in the event the car is currently in an intersection, it will commence turning upon arriving at the next traffic light on the current road it is on. This restriction is based on the reality that a car in the middle of an intersection can not make a decision to turn without updating its velocity prior to arriving at the intersection. The Intersection Flag is used to denote cars that are in the intersection at which they are scheduled to turn. Secondly, consider $s_{n} \geq v_{\text {turn }}$. If the traffic signal is red then the vehicle proceeds as in the turn-free model, $v_{n} \rightarrow \min \left(v_{n}, d_{n}-1, s_{n}-1\right)$. When the traffic signal is green the vehicle will not be able to complete the turn at the current time-step and, at best, will become situated in the intersection itself with speed $s_{n}$. At the next time step the car will complete its turn with a velocity of $\min \left(v_{n}, v_{\text {turn }}, d_{n}-1, s_{n}-1\right)$. In this situation it should be made clear that no vehicle will be selected to turn again until it completes the selected turn.

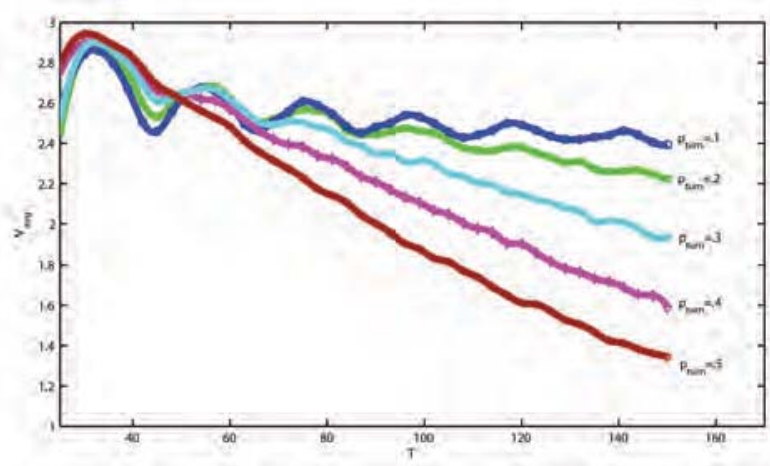

Figure 3. The average velocity is plotted versus the light cycle length for $p_{\text {slow }}=.1$, various turning probabilities, and $d_{x}=d_{y}=101$ for $\rho=.05$. Each point resembles the average velocity after aggregating the model for 125,000 steps.

Lastly, consider $s_{n}<v_{\text {turn }}$. In this situation the vehicle is close enough to the intersection to complete the turn in one time step provided $s_{n}<\min \left(v_{n}, v_{\text {turn }}, d_{n}-1, \widetilde{d}_{n}-1\right)$. The Turning Flag is turned on when a car is making a turn in a single time step, and must be moved onto the cross street in step 4 with special considerations. The position of an eastbound car turning northbound will be $a_{i j} \rightarrow a_{\mu v}$ where $\mu=i+s_{n}$ and $v=j-\left(v_{n}-s_{n}\right)$, where sn is the distance to the turning intersection. Similarly for a northbound car turning eastbound $\mu=i+\left(v_{n}-s_{n}\right)$ and $v=j+s_{n}$. In no situation is turning allowed when the light is currently red.

A vehicle will enter the intersection only if there is room for it. This modification over the BML model was introduced ${ }^{5}$ to attenuate the transition to a grid-locked state by restricting movement into the intersection, hence preventing blockage of the perpendicular lane of traffic.

In step 3 the vehicles randomly brake with randomization parameter $p_{\text {slow }}$. This models the effect of random slowing due to different behavioral patterns of drivers. Updating the position of each car is done in step 4. 
The Nagel-Schreckenberg model ${ }^{12}$ can be realized by setting $p_{\text {slow }}=0, N_{x}=1$, and $N_{y}=0$. Upon removal of $(a)$ in the second step and setting $p_{\text {turn }}=0$ the model reduces to Brockfeld et al.'s model. ${ }^{5}$ Adding turning to a basic CA traffic model allows us to better analyze the specific effect of turning on the flow of traffic. The influence of the new parameter $p_{\text {turn }}$ is addressed in the following section.

\begin{tabular}{|c|r|r|r|r|}
\hline$\epsilon / \rho$ & .05 & .10 & .15 & .20 \\
\hline .1 & $(444.537,-0.892)$ & $(401.091,-0.997)$ & $(403.052,-0.997)$ & $(513.239,-.943)$ \\
\hline .2 & $(217.572,-0.898)$ & $(231.090,-.881)$ & $(203.920,-.988)$ & $(250.989,-.997)$ \\
\hline .3 & $(141.552,-.857)$ & $(142.404,-.846)$ & $(134.436,-0.992)$ & $(141.059,-.877)$ \\
\hline .4 & $(106.427,-.860)$ & $(106.427,-.860)$ & $(101.720,-0.983)$ & $(104.620,-.945)$ \\
\hline .5 & $(85.853,-.898)$ & $(86.266,-.855)$ & $(81.759,-.952)$ & $(78.386,-.945)$ \\
\hline
\end{tabular}

Table 1. $(K, \eta)$ Values

It is reasonable to expect that introducing turning will reduce the average velocity $V_{\text {avg }}$ compared with the turn-free situation. In essence a vehicle selected for turning behaves similar to that of one approaching a red light, slowing down as the vehicle approaches it. Theoretically this is easily realized. Consider $p_{\text {turn }}=p_{\text {slow }}=0$, it is straight-forward to verify that

$$
\lim _{T \rightarrow \infty} V_{\text {avg }}=\min \left(\frac{1-\rho}{2 \rho}, \frac{v_{\max }}{2}\right):=V_{\text {steady }}
$$

This is in contrast to the case when $p_{\text {turn }}>0$ as for sufficiently long enough light cycles all the cars will eventually turn on the perpendicular road and come to a stop at the next red light. In other words for $p_{t u r n}>0$ the $\lim _{T \rightarrow \infty} V_{a v g}=0$. This is indicative of a critical light cycle length for which the average velocity may decrease sharply. In either case, it is suggestive that one may find a critical length cycle $T_{\epsilon}$, such that $V_{\text {avg }}<\epsilon V_{\text {steady }}$ for a prescribed $\epsilon>0$. In other words, one may determine the light cycle length that will only lower the average speed by $50 \%$ compared to the steady-state, turn-free, velocity. This motivates our exploration into a power law that depends on $\rho$ and $p_{\text {turn }}$ for a prescribed $\epsilon$. 


\section{RESULTS - A POWER LAW}

In the given calculations the simplest network is investigated with only one east and north bound intersection with periodic boundary conditions. The turning velocity is held constant at 1 . The maximum velocity $v_{\max }$ is set to 5 . The length of the road is 101 cells, i.e. $d_{x}=d_{x}=100$. For each value of the light cycle length, density, and probabilities of slowing and turning the model is aggregated for 125,000 time steps. Upon completion the average velocity is calculated by averaging the the calculated velocities at each time-step. A typical plot for $p_{\text {slow }}=.1$ and $\rho=.05$ is shown in Fig. 3 .

The value of $p_{\text {turn }}$ has a considerable influence on the average velocity for each simulation. For extremely small probabilities the average velocity has the same fundamental characteristics as in the $p_{\text {turn }}=0$ case (see Fig. 3). At large densities $\rho>.5$ jamming can occur provided the light cycle length is long enough and $p_{\text {turn }}>0$. For illustration let the light be green for eastbound(northbound) traffic and $p_{\text {slow }}=0$. Turning vehicles onto the northbound(eastbound) lane will be forced to slow down as a result of the upcoming traffic light. If the light cycle is long enough the vehicles will aggregate to the northbound(eastbound) lane. This will occur when the light cycle is longer than the number of time-steps it takes for all the eastbound( northbound) cars to be selected for turning $\left(\frac{1}{p_{u u m}}\right)$ and complete the turn at the next intersection, i.e.

$$
T>T^{*}=\frac{1}{p_{\text {turn }}}+\min \left(d_{x}, d_{y}\right) . \quad \text { Equation } 1 .
$$

For $T>T^{*}$ and $\rho>.5$ the northbound (eastbound) lane will come to a complete stop preventing eastbound (northbound) cars from moving through the intersection. The jam will persist till the light changes and the car blocking the intersection is selected again for turning. In reality for even small values of $p_{\text {turn }}>0$ and $\rho$ is low as . 2 jamming may occur for $T<<T^{*}$. It is reasonable to compare the observed jamming to real-life situations when a car sits in the intersection as a result of heavy traffic consequently interfering the perpendicular flow of traffic. This may suggest that for heavy traffic long light cycle lengths $\left(T>T^{*}\right)$ should be avoided as a deterrent to the formation of jams. The persistence of jamming appearing at densities as low as .2 suggests that a synchronous lighting strategy may not be ideal outside of low density traffic.

To avoid jamming caused by the introduction of turning, densities less than .2 will be considered only. In addition, to focus exclusively on the effect of turning, $p_{\text {slow }}$ will be set to zero in the following calculations. The algorithm is simulated at different densities and values of $p_{\text {turn }}$. $v_{\text {turn }}$ remains at one, and $v_{\max }=5$. The amplitude of the oscillations observed in the turnfree case are reduced and vanish as the turning probability increases (see Fig. 3). The light cycle length, $T_{\epsilon}$, for which the average velocity is first less than, $\epsilon V_{\text {steady }}$ is determined. It is clear that this satisfies a power law distribution that depends on $\rho$ and $p_{\text {turn }}$ (see Fig. 4 for the $\rho=.1$ case). More precisely,

$$
T_{\epsilon}\left(p_{\text {turn }}, \rho ; \epsilon\right)=K p_{\text {turn }}^{\eta}, \quad \text { Equation } 2 .
$$




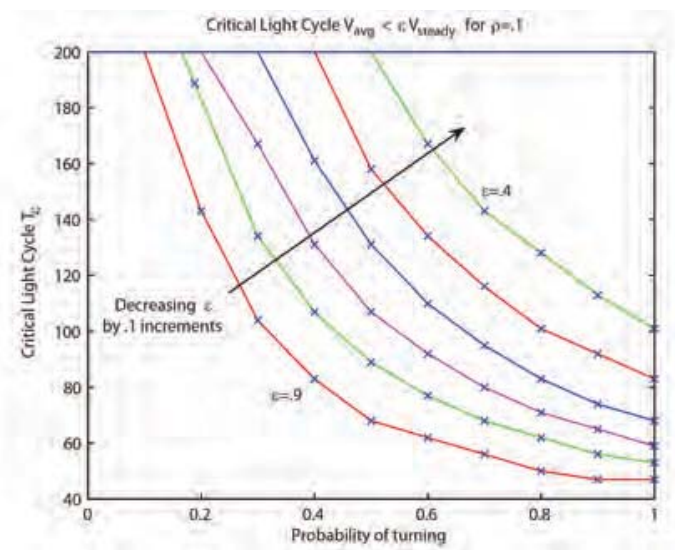

Figure 4. $T_{\epsilon}$ is calculated numerically for $\epsilon=.5, \rho=.1, d_{x}=d_{y}=101$, and $p_{\text {slow }}=0$.

The value of $p_{\text {turn }}=.1, .2, \ldots, 1$.

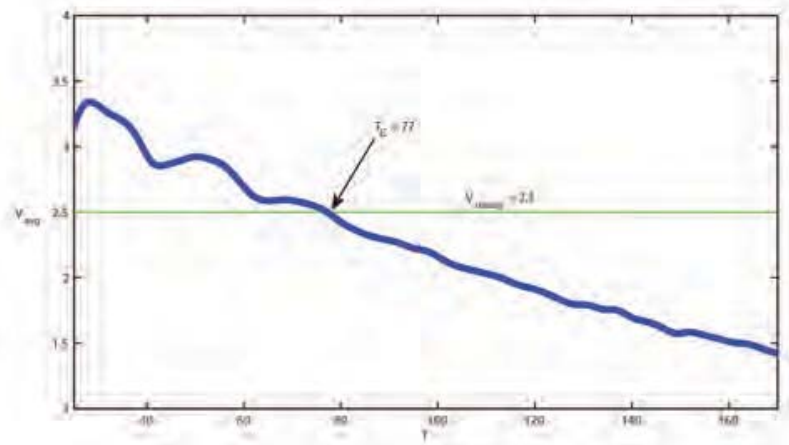

Figure 5. $T_{\epsilon}$ is shown for $\epsilon=1, \rho=.05, d_{x}=d_{y}=101, p_{\text {turn }}=.4$, and $p_{\text {slow }}=0$.

Notice for values $T>T_{\epsilon}$ the average velocity continues to decrease.

The experimental value of $T_{\epsilon}$ is 77 . Using Eq. 3 predicts a value of 75.67 .

where $K$ and $\eta$ are functions that depend on $\rho$ and $\epsilon$. The $(K, \eta)$ coordinate pair for $\rho=.05, .1, .15$, and .2 are shown in Table I. The function $K$ also obeys a power law of the form $K_{0}(\rho) \epsilon^{\eta 0(\rho)}$. The function $\eta$ is fitted to a plane in a least squares sense. This results in a more general power law

$$
T_{\epsilon}\left(p_{\text {turn }}, \rho ; \epsilon\right)=\left(m_{k} \rho+b_{k}\right) \epsilon^{m \eta \rho+b \eta} p_{\text {turn }}^{a \epsilon+b \rho+c}
$$

Equation 3.

where $m_{k}, b_{k}, m_{\eta}, b_{\eta}, a, b$, and $c$ are constants are shown in Table II.

\begin{tabular}{|c|r|}
\hline$m_{k}$ & -24.76345201312850 \\
\hline$b_{k}$ & 42.89042951253280 \\
\hline$m_{\eta}$ & -0.50559741111383 \\
\hline$b_{\eta}$ & -0.98871563734671 \\
\hline$a$ & 0.06225834560947 \\
\hline$b$ & -0.22611688813737 \\
\hline$c$ & -0.88937638486533 \\
\hline
\end{tabular}

Table 2. Least Squares Coefficients for Eq. 3 
As an example, Fig. 5 shows the average velocity for various light cycle lengths. In the case of $\epsilon=1$ the average velocity is less than $V_{\text {steady }}$ once $T>77$. Using Eq. 2 and the coefficients found in Table I the predicted value is 75.67. Clearly, these are comparable results.

Fig. 6 shows $\epsilon$-slices as predicted from the theory for $\rho=.05$ compared with experimental results. For example, for a $40 \%$ turning probability Eq. 3 predicts that light cycle lengths greater than 200 will generate a $50 \%$ reduction in the average velocity.

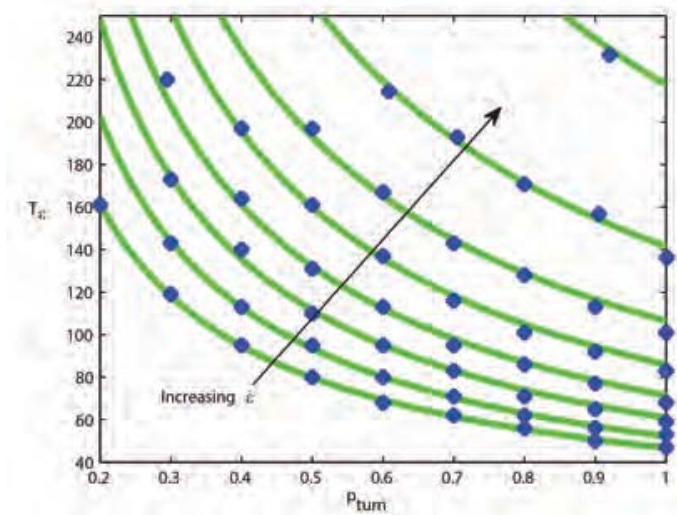

Figure 6. Experimental computation of $T_{\epsilon}$ versus Eq. 3 for $\rho=.05$ and $\epsilon=.9, \ldots, .1$. The maximum least squares error is 0.0096228 clearly showing good agreement.

\section{DISCUSSION}

A simple model for traffic flow was introduced borrowing ideas from previous models. ${ }^{5}$ It is shown that persistent jamming can occur as a result of turning, even for densities that are rather low. In fact, jamming is guaranteed for moderate densities with sufficient light cycle lengths. Therefore it is a synchronous lighting strategy which should be avoided in these situations.

In the analysis of lower densities it was shown that the average velocity is reduced by cars turning onto cross streets. In particular, it was shown that a power law depending on the density and the probability of turning is found that can predict when the average velocity is lower than $\epsilon V_{\text {steady }}$ for all $T>T_{\epsilon}$. It is clear that a traffic controller using a synchronous lighting strategy should chose a light cycle length sufficiently less than $T_{\epsilon}$ to avoid large reductions in the flow of traffic. 


\section{REFERENCES}

[1] Aw, A., and Rascle, M. (2000) Resurrection of "second order" models of traffic flow, SIAM Journal on Applied Mathematics 60, 916-938.

[2] Bellomo, N., Delitala, M., and Coscia, V. (2002) On the mathematical theory of vehicular traffic flow i. fluid dynamic and kinetic modelling, Mathematical Models and Methods in Applied Sciences 12, 1801-1843.

[3] Biham, O., Middleton, A. A., and Levine, D. (1992) Self-organization and a dynamical transition in traffic-flow models, Phys Rev A 46, R6124-R6127.

[4] Brenneis, A., Konhauser, P., and Kerner, B. (1995) Comparison of one and two equation models for traffic flow calculations, In In Proc. 26th AIAA Fluid Dynamics Conference, San Diego, CA.

[5] Brockfeld, E., Barlovic, R., Schadschneider, A., and Schreckenberg, M. (2001) Optimizing traffic lights in a cellular automaton model for city traffic, Phys Rev E Stat Nonlin Soft Matter Phys 64, 056132.

[6] Chowdhury, D., Santen, L., and Schadschneider, A. (2000) Simulation of vehicular traffic: a statistical physics perspective, Computing in Science and Engineering and Science 2, 80-87.

[7] Chowdhury, D., Santen, L., and Schadschneider, A. (2000) Statistical physics of vehicular traffic and some related systems, Physics Reports 329.

[8] NH, G., and Stamatiadis, C. (2009) Traffic networks, optimization and control of urban, In Encyclopedia of Complexity and Systems Science, pp 9470-9500.

[9] Helbing, D. (1995) Improved fluid-dynamic model for vehicular traffic, Phys Rev E Stat Phys Plasmas Fluids Relat Interdiscip Topics 51, 3164-3169.

[10] Kerner, B. (2004) The Physics of Traffic, Springer.

[11] Klar, A., Kuhne, R., and Wegener, R. (1996) Mathematical models for vehicular traffic, Surveys Math. Indust 6, 215-239.

[12] Nagel, K., and Schreckenberg, M. (1992) A cellular automaton model for freeway traffic, J Phys I 2, 2221-2229.

[13] Payne, H. (1971) Models of freeway traffic and control, Math. Models Publ. Sys 1, 51-61.

[14] Prigogine, I. (1961) Theory of traffic flow, A Boltzman like approach to the statistical theory of traffic flow, Elsevier Publishing Company.

\section{ABOUT THE STUDENT AUTHOR}

Tracy Finner worked on this project during her senior year (2010-2011) at the University of Arizona. She finished her M.Sc. in industrial engineering in the spring of 2014. She is now an Industrial Engineer at Raytheon.

\section{PRESS SUMMARY}

Traffic flow models are central to urban planners and developers. In this paper, a traffic flow model is proposed that models the interaction between individual vehicles and subsequent driver behavior to the traffic light system. The model is then used to develop empirical evidence for a statistical correlation between the density of vehicles, frequency of turning vehicles, and the timing of the traffic signal. 